\title{
Unaffected family members report improvements in daily routine sun protection 2 years following melanoma genetic testing
}

\author{
Lisa G. Aspinwall, PhD', Jennifer M. Taber, PhD', Wendy Kohlmann, MS, CGC'2, Samantha L. Leaf, PhD' \\ and Sancy A. Leachman, MD, $\mathrm{PhD}^{3}$
}

Purpose: Reducing ultraviolet radiation exposure may decrease melanoma risk in the hereditary melanoma setting. It is unknown whether genetic counseling and test reporting of $C D K N 2 A / p 16$ mutation status promote long-term compliance with photoprotection recommendations, especially in unaffected mutation carriers.

Methods: This study evaluated changes 2 years following melanoma genetic testing in self-reported practice of sun protection (sunscreen, photoprotective clothing, and ultraviolet radiation avoidance) among 37 members of two $C D K N 2 A / p 16$ kindreds (10 unaffected carriers, 11 affected carriers, and 16 unaffected noncarriers; response rate = 64.9\% of eligible participants).

Results: Multivariate profile analysis indicated that all three participant groups reported increased daily routine practice of sun protection 2 years following melanoma genetic testing $(P<0.02)$, with $96.9 \%$ reporting that at least one sun protection behavior was part of their daily routine, up from $78.1 \%$ at baseline $(P<0.015)$. Unaffected carriers $(P<0.024)$ and unaffected noncarriers $(P<0.027)$ reported significantly more frequent use of photoprotective clothing. Affected carriers maintained adherence to all sun protection behaviors. Reported sunburns in the past 6 months decreased significantly $(P<0.018)$.

Conclusion: Members of high-risk families reported increased daily routine sun protection and decreased sunburns 2 years following melanoma genetic testing, with no net decline in sun protection following negative test results. Thus, genetic testing and counseling may motivate sustained improvements in prevention behaviors.

Genet Med advance online publication 24 April 2014

Key Words: CDKN2A/p16; genetic testing; melanoma prevention; sunburn; sun protection

\section{INTRODUCTION}

The goal of predictive genetic testing is to alert people to elevated risk prior to disease onset, when early detection and prevention may still be possible. Little is known about the impact of genetic test reporting on cancer prevention behaviors, as most research has focused on hereditary cancers for which genetic testing promotes increased screening and/or prophylactic surgery (i.e., breast and colon cancers). ${ }^{1,2}$ Determining whether genetic and genomic information improves prevention behaviors is a major priority for communication and social sciences research. ${ }^{3-6}$ For melanoma, the genetic risk conferred by a CDKN2A/p16 (or simply, p16) mutation may interact with personal behavior (specifically, exposure to ultraviolet radiation (UVR)) to determine risk. Thus, studying responses to genetic testing and counseling among melanoma-prone families provides a unique model for testing whether genetic test reporting improves compliance with prevention recommendations. Mutations in the p16 tumor suppressor account for 20 to 40\% of high-risk melanoma families and confer up to a $76 \%$ lifetime risk. ${ }^{7}$ Population-based studies have found a lower, but still significant, risk of $28 \%$ by the age of 80 years. ${ }^{8}$ The single best characterized environmental risk factor for melanoma is UVR, and epidemiologic studies suggest that UVR superimposes additional melanoma risk on high-risk families. ${ }^{7}$ Consistent practice of sun protection behaviors may help individuals who carry $p 16$ mutations to minimize the risk associated with their inherited mutation.

Although melanoma genetic testing is transitioning into the clinical arena, ${ }^{9}$ this transition has been slow due to questions regarding whether genetic testing improves medical management or adherence for people who are already known to need photoprotection and screening based on family history. ${ }^{10,11}$ Also, management recommendations concerning prevention may not differ for mutation carriers and noncarriers. Clinically identified $p 16$ families often also have additional risk factors such as a melanoma-prone phenotype and/or history of UVR exposure. Therefore, relatives testing negative for familial mutations may still be at increased risk for melanoma. For these reasons, it is important to examine (i) whether positive genetic test results promote long-term improvements in prevention behaviors and (ii) whether negative results promote decreased adherence. 
A strong family history of melanoma does not appear to sufficiently motivate appropriate adherence to sun protection behaviors. Unaffected members of high-risk families (those without a personal history of melanoma) report significantly less frequent practice of sun protection behaviors than their affected counterparts and more frequent risk behaviors (e.g., sunbathing, tanning bed use, and sunburns). ${ }^{12-14}$ Studies of high-risk families suggest a strong interest in genetic testing for melanoma, largely driven by the belief that a positive test result would motivate improved sun protection. ${ }^{15-19}$ The few studies of the impact of melanoma genetic testing on sun protection behaviors have included predominantly affected participants $\mathrm{s}^{20,21}$ and have shown little improvement to their already high levels of sun protection. ${ }^{17}$

It remains an open question whether unaffected family members change their photoprotection behavior following genetic testing. Because unaffected relatives are less compliant than family members with a melanoma history, they may benefit more from interventions. Our initial short-term follow-up study of p16 test reporting ${ }^{12}$ indicated that unaffected carriers increased intentions to practice all three recommended methods of photoprotection (sunscreen use, photoprotective clothing, and UVR avoidance) and reported somewhat more frequent sun protection at 1 month after test reporting. Those who received negative test results also reported increased behavioral intentions, with no net decline in overall photoprotection at 1 month. The present study extends this earlier report by examining follow-up data from the same sample to assess whether these intended improvements in the consistent practice of sun protection behaviors were implemented and practiced 2 years later, as sustained behavior change is required to significantly moderate melanoma risk conferred by genetic factors.

\section{MATERIALS AND METHODS \\ Study population and procedures}

Companion test reporting and follow-up studies were approved by the Institutional Review Board at the University of Utah (IRB nos. 7916 and 13816). Participants recruited for this study were adult members of two large melanoma pedigrees who had contributed DNA samples for prior melanoma genetic research, beginning with an institutional review board-approved study in the late 1980s that utilized the Utah Population Database to identify pedigrees with a hereditary pattern of melanoma. ${ }^{22,23}$ These studies contributed to the identification of the p16 loci; specifically, two kindreds were found to have deleterious p16 mutations (V126D and $5^{\prime} \mathrm{UTR} 34 \mathrm{G}>\mathrm{T}$ ). In the early 2000s, every living participant in the gene identification studies was invited to participate in a phenotyping study, which included detailed phenotype characterization and mutation testing, but no participants were aware of the presence of the p16 mutation in their family or their personal mutation status. ${ }^{24}$ During the phenotyping study, participants received an individual education session during which they were informed that they were part of a high-risk family and provided with detailed recommendations for photoprotection and screening.
For the purposes of the present study, every participant in the phenotyping study who was a member of a p16-positive kindred $(n=77)$ was recontacted and invited to participate in the present study (see ref. 25). Recruitment and retention are summarized in Figure 1. Not included in the figure or the retention statistics are 3 of the 77 participants, who had a history of melanoma but tested negative for the p16 mutation. These participants completed multiple follow-up assessments but were excluded from the analysis because there were too few to permit meaningful comparisons. From May through November 2005, 61 (82.4\%) participants completed a written baseline questionnaire immediately before a genetic counseling session that included the option to receive individual p16 results, followed by test reporting. All 61 participants elected to receive results during the counseling session. After completing a post-session questionnaire, 57 participants were invited to enroll in the companion follow-up study of long-term psychological and behavioral responses to $p 16$ test reporting. ${ }^{26,27}$ As shown in Figure 1, 51 participants (89.5\% of those eligible) were enrolled. Participants received modest nonmonetary incentives (e.g., water bottles and tote bags) for completing follow-up questionnaires. Results of short-term analyses were previously reported. ${ }^{12}$ As shown in Figure 1, 37 participants (10 unaffected carriers, 11 affected carriers, and 16 unaffected noncarriers; $64.9 \%$ response rate) completed the 2-year follow-up

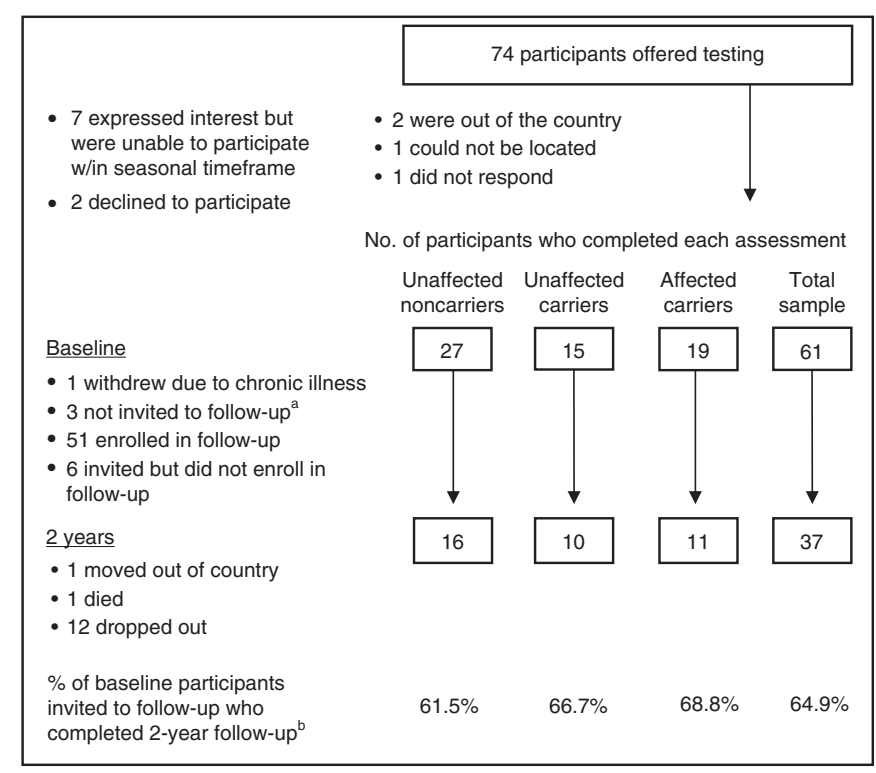

${ }^{a}$ One began study participation after the seasonal cutoff date (an eligibility criterion intended to provide seasonal adjustment for measures of

photoprotection). The other two were not invited to the follow-up study

because one participant was adopted and the other was from a small kindred. Given the focus on family communication and other familial issues in other parts of the questionnaire, it was decided that these participants' experience with genetic counseling and test reporting would be substantially different from the experiences of other participants.

${ }^{\mathrm{b}}$ Retention rates for the 2-year follow-up assessment were calculated out of the 57 eligible participants (26 unaffected noncarriers, 15 unaffected carriers, and 16 affected carriers) who were invited to the follow-up study.

Figure 1 Recruitment, retention, and attrition of noncarriers, unaffected carriers, and affected carriers at baseline and 2-year follow-up. 
assessment; of these, 5 participants ( 3 unaffected noncarriers and 2 unaffected carriers) completed an abbreviated phone version.

\section{Pre- and post-disclosure genetic education and counseling} Research tests were confirmed in a Clinical Laboratory Improvement Amendments-certified laboratory. Genetic counseling and test reporting were conducted in a single session. ${ }^{25}$ Melanoma risk associated with $p 16$ mutations was presented as a $35-$ to 70 -fold increase from general population risk $(\sim 1 \%)$ and illustrated with a bar graph indicating a risk of $50 \%$ by the age of 50 years and $76 \%$ by the age of 80 years. After receiving genetics education and providing written informed consent, all 61 participants agreed to receive their genetic test results. Those testing negative for the familial mutation were informed that they still may have up to a 1.7 -fold residual risk due to other co-inherited familial risks such as melanoma-prone phenotype and UVR exposure. All participants were advised to manage their cancer risk by minimizing sun exposure and preventing sunburns. Specifically, participants were advised to avoid the sun between $10 \mathrm{AM}$ and $4 \mathrm{PM}$, to seek shade, to use sunblock with sun protection factor $30+$ with reapplication every 2 hours, and to wear protective clothing. A letter was sent about 1 month later to reinforce these management recommendations.

\section{Measures}

Demographic information and medical history. Participants completed standard demographic questions and indicated whether a medical professional usually cared for their skin. Melanoma history at baseline was confirmed through medical records. ${ }^{25}$ Participants were asked to self-report any new melanoma diagnoses in themselves or their family members during the course of the study. These reports were confirmed through the Utah Cancer Registry.

Frequency of sun protection behaviors. At baseline and 2 years, participants reported the percentage of time they used sunscreen, wore protective clothing, avoided peak UVR exposure from $10 \mathrm{AM}$ to $4 \mathrm{PM}$, and stayed in the shade in the past 6 months. Seven response options ranged from "never used-about $0 \%$ of the time" to used "all the time-about $100 \%$ of the time." Each response option paired verbal and numeric descriptors (e.g., "occasionally" with "...30-40\% of the time").

Daily routine practice of sun protection behavior. At baseline and 2 years, participants rated the extent to which each sun protection behavior (sunscreen, photoprotective clothing, and UVR avoidance) was part of their daily routine $(1=$ not at all; $5=$ very much). Because the abbreviated phone survey completed by five participants at follow-up assessed frequency but not daily routine practice of sun protection behavior, the effective sample size for these daily routine measures was 32 .

Reports of sunburns, sunbathing, and tanning booth use. At baseline and at 2 years, participants indicated how many sunburns they received in the past 6 months, ranging from none to more than 2-3 per week. Participants also reported if they had sunbathed (spent time in the sun with the purpose of getting a tan) or used a tanning bed in the past 6 months, and, if so, how often (from 3 or more times per week to once per month). Answers were converted to the number of sunbathing and tanning occasions and sunburns in the past 6 months. Additionally, because of the importance of sunburns in the etiology of melanoma, ${ }^{28}$ participants who reported sunburns at the 2-year follow-up were asked to describe how they became sunburned. Written responses were coded by two raters, with $84.2 \%$ agreement.

\section{OVERVIEW OF DATA ANALYSIS}

Changes in sun protection and risk behaviors following melanoma genetic test reporting were evaluated first by examining changes in the reported frequency and daily routine practice of multiple methods of sun protection behavior over time in the three participant groups. Because the four major recommended photoprotection behaviors functionally overlap and were only weakly intercorrelated, it was not desirable to create an overall photoprotection index by averaging them. Accordingly, multivariate profile analyses were conducted to examine changes from baseline to 2 years in the patterning and frequency of overall photoprotection behavior. ${ }^{12,29}$ Planned contrasts evaluating change over time in the specific patient groups used onetailed tests of significance with hypotheses in the direction of improved sun protection. Second, we examined changes from baseline to 2 years in risk behaviors (sunbathing, tanning, and sunburns). To better understand failures of sun protection efforts in high-risk participants, we also examined participantidentified reasons for sustaining sunburns. Last, we examined the relation of personal and familial melanoma diagnoses during the study to reported prevention behaviors.

\section{Participant characteristics}

\section{RESULTS}

The average age of participants was 45.2 years $(S D=14.65)$. All were white, $54.1 \%$ were male, $86.5 \%$ were married, $91.9 \%$ had a high school education or higher, and the median income was $\$ 60,000-\$ 69,000$. All but three participants lived in Utah for the duration of the study. Ninety percent of unaffected carriers, $100 \%$ of affected carriers, and $62.5 \%$ of noncarriers reported having a medical professional (almost always a dermatologist) who normally cared for their skin. Participants with a melanoma history $(n=11)$ had an average of $2.73(\mathrm{SD}=2.45)$ past melanomas. There were no significant differences in age, sex, education, or income among the three participant groups. Furthermore, there was no evidence of differential attrition among the three groups at 2 years, and participants who did not complete the 2-year assessment did not differ on any demographic variable (age, sex, education, or income), baseline sun protection behavior, sunburns, or tanning booth use from those who provided follow-up data. Medical follow-up data indicated that three participants, all with a personal history of melanoma 
at baseline, reported either a recurrence or one or more new melanomas, and 10 participants ( 1 noncarrier, 4 unaffected carriers, and 5 affected carriers) reported that a family member was diagnosed with melanoma during the study.

\section{Changes in sun protection behavior over the 2-year period} Percentage of time the multiple methods of sun protection were used in the past 6 months. A repeated-measures analysis was conducted across the profile of four sun protection behaviors to examine changes from baseline to 2 years following melanoma genetic test reporting in the three participant groups. This analysis yielded three significant effects. First, as shown in Figure 2, a significant main effect of group indicated that the profiles of sun protection behavior were different in the three groups $(F(8,62)=2.155 ; P<0.043)$, such that affected carriers reported high levels of all three behaviors, whereas unaffected family members showed more variation. Second, the only significant difference among the three participant groups in an individual sun protection behavior was that affected carriers

a



b

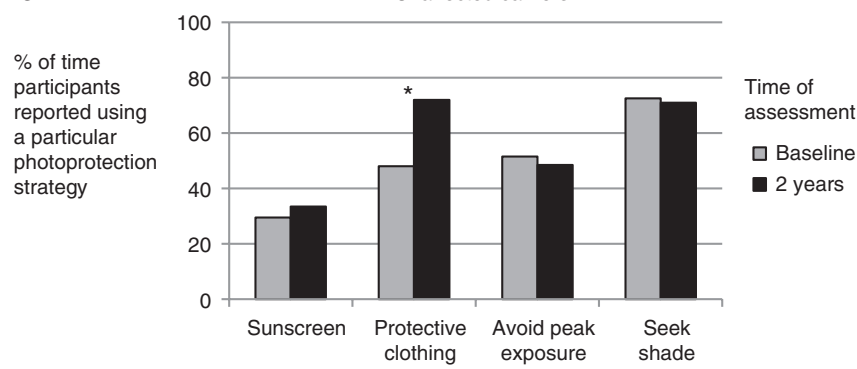

C

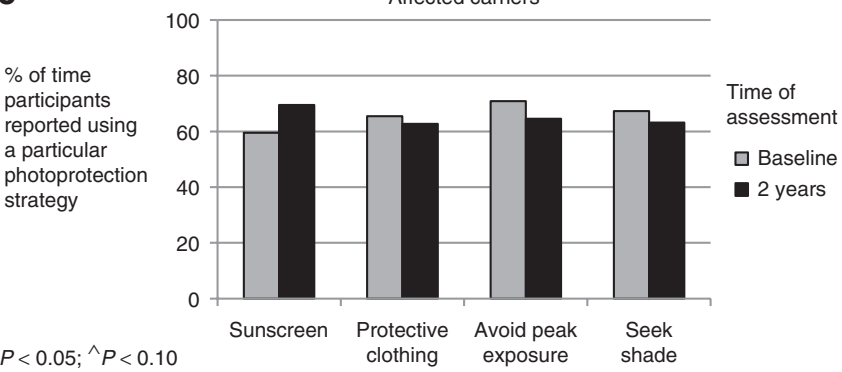

Figure 2 Percentage of time in the past 6 months that participants reported using each of the recommended sun protection behaviors (using sunscreen, wearing protective clothing, avoiding peak UVR exposure, seeking shade) at baseline and 2 years following melanoma genetic test reporting for (a) unaffected noncarriers, (b) unaffected carriers, and (c) affected carriers. UVR, ultraviolet radiation. reported more frequent sunscreen use at both assessments (64.55\%) than unaffected carriers $(31.50 \% ; P<0.003)$, and somewhat more than unaffected noncarriers $(47.97 \%$; $P<$ 0.085; main effect of group; $F(2,34)=5.027 ; P<0.012)$. Third, although there were no significant net changes over time across the profiles of behavior in any participant group $(F(4,31)=1.832$; $P<0.148$ ), reported use of photoprotective clothing increased significantly over time (from $53.65 \%$ of the time at baseline to $67.30 \%$ at 2 years; $F(1,34)=4.546 ; P<0.040)$. Follow-up tests indicated that photoprotective clothing use reliably increased among both unaffected noncarriers (from 49.06 to $67.50 \%$ of the time; $P<0.027$; Figure 2a) and unaffected carriers (from 48 to $72 \% ; P<0.024$; Figure $2 \mathbf{b}$ ), with no change among affected carriers (65.46-62.73\%; $P<0.404$; Figure 2c). There were no significant changes in reported frequency of sunscreen use, avoidance of peak exposure, or shade seeking in any participant group.

Daily routine practice of multiple methods of sun protection. We next examined change from baseline to 2 years following test reporting in the extent to which participants characterized each of the three strategies as part of their daily routine. The multivariate profile analysis yielded a significant overall increase across the three strategies for all participant groups (main effect of time: $F(3,27)=6.575$; $P<0.002$; Figure 3). Follow-up tests indicated significant increases across the profile of sun protection behaviors among unaffected noncarriers $(F(1,12)=18.353 ; P<0.001$; Figure 3a) and unaffected carriers $(F(1,7)=10.938 ; P<0.013$; Figure $3 \mathbf{b})$ but no net increase among affected carriers $(F(1,10)=1.720$; $P<0.219$; Figure 3c). As was the case with the behavioral frequency data, reported daily routine sunscreen use tended to be higher among affected carriers than other groups $(F(2,29)$ $=2.620 ; P<0.090)$.

Daily routine practice of one or more sun protection methods at 2 years. We next examined changes over time in the proportion of participants reporting moderate or high levels of daily routine practice of one or more sun protection methods. The proportion reporting adherence to at least one such method increased significantly from $78.1 \%$ at baseline to $96.9 \%$ at 2 years (McNemar exact test, $P<0.015)$. With nearly universal compliance to at least one recommended sun protection method at 2 years (only one participant, an affected carrier, did not report daily routine practice of any sun protection method), there were no differences among the participant groups at follow-up.

\section{Changes in risk behaviors over the 2-year period}

Sunburns. A substantial proportion of participants reported one or more sunburns in the past 6 months at both assessments ( $50 \%$ at baseline and $41.7 \%$ at 2 years). However, the average number of sunburns decreased significantly from 1.29 at baseline to 0.56 at 2 years (main effect of time: $F(1,33)=$ $6.22 ; P<0.018$ ), with no differences by group (time $\times$ group 


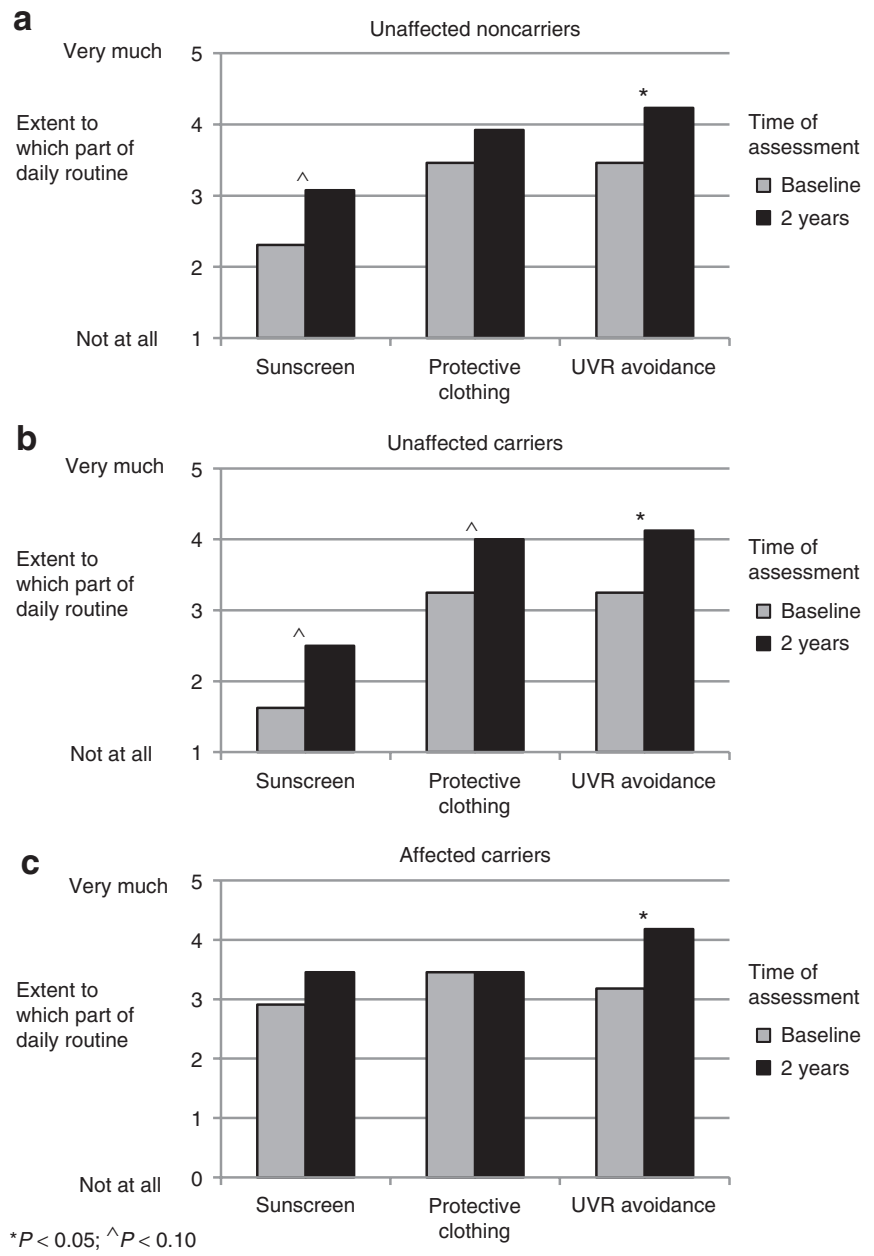

Figure 3 Reported daily routine use of sunscreen, protective clothing, and UVR avoidance at baseline and 2 years following melanoma genetic test reporting for (a) unaffected noncarriers, (b) unaffected carriers, and (c) affected carriers. UVR, ultraviolet radiation.

interaction: $F(2,33)=1.07 ; P<0.354)$. Simple-effects tests indicated that the decrease over time was significant only for affected carriers $(P<0.026)$, whose sunburns decreased from an average of 2.01 at baseline to 0.60 at 2 years. Additional inspection of the data indicated that two participants had outlying sunburn scores at baseline that were $>3$ SDs from the sample mean (an affected carrier with 12 and an unaffected carrier with 6). Both participants reported two sunburns at 2 years. With these outliers excluded from the analysis, the decline in sunburns following test reporting in the overall sample remained significant ( 0.83 sunburns at baseline and 0.47 at 2 years; main effect of time: $F(1,31)=5.02 ; P<0.032$ ).

Sunbathing. Only two participants, both unaffected carriers, reported sunbathing at baseline. At 2 years, neither reported sunbathing in the past 6 months.

Tanning bed use. At baseline, tanning bed use was reported by one unaffected carrier (who did not report frequency) and one affected carrier (at a frequency of once every 2 weeks). At
2 years, only the same affected carrier reported still using a tanning bed, at the same frequency.

Reasons for sunburns. Because nearly all participants reported daily routine practice of at least one sun protection method but $41.7 \%$ nonetheless reported sustaining sunburns at followup, we examined participants' accounts of how they came to be sunburned. All 15 participants who reported at least one sunburn at 2 years provided an explanation (two provided $>1$ response). The most common response, provided by nearly half of those reporting sunburns, indicated difficulties with sunscreen use-specifically, four participants (26.7\%) reported forgetting sunscreen or missing a spot and four participants $(26.7 \%)$ reported that their sunscreen wore off during exercise or water activities (e.g., "Went to water park. I used sunscreen $60+$ but still got burned after only 2 hours. Non-peak hours of 4:00-6:00."). Three (20.0\%) participants reported becoming burned because they were outside too long (e.g., "Accidentally stayed out too long and ran out of sunscreen"), and one participant (6.7\%) each reported unanticipated sun exposure, insufficient available shade, tanning bed use, and becoming burned despite trying to seek shade. Two participants did not specify the reason (i.e., "lack in precautions" and "accidentally on arms and legs"). These responses highlight some of the difficulties in managing consistent daily sun protection as even participants who used sunscreen and deliberately avoided peak hours or sought shade reported sunburns.

\section{Relation of personal and familial melanoma diagnoses during the study to prevention behaviors at 2 years}

We examined personal and familial melanoma diagnosis during the course of the study as predictors of sun protection and risk behaviors at 2 years. These analyses are exploratory due to the low sample sizes in each subgroup. The three participants who developed a new melanoma did not account for affected carriers' high level of adherence: on average, they were significantly less adherent at 2 years on measures of daily UVR avoidance (3.00 vs. $4.63 ; P<0.005)$ and reported more sunburns $(1.50$ vs. $0.38 ; P<0.031)$ than other affected carriers. Participants who reported that a family member was diagnosed with melanoma during the study ( $40 \%$ of unaffected carriers and $45.5 \%$ of affected carriers) did not differ significantly in any sun protection or risk behavior at 2 years from participants in their respective groups who reported no new diagnoses of melanoma in the family.

\section{DISCUSSION}

Two years following melanoma genetic test reporting and counseling, both groups of unaffected family members, those found to carry a $p 16$ mutation and those receiving negative test results, reported sustained improvements in sun protection behavior, notably in the frequency of photoprotective clothing use and in the daily routine practice of sun protection behaviors. These findings are especially important for unaffected carriers, who are at highly elevated genetic risk but do not have 
a personal history of melanoma to motivate appropriate prevention behaviors. Affected carriers continued to report a high degree of adherence across the profile of recommended sun protection behaviors. Importantly, at 2 years, nearly all participants reported daily routine practice of at least one sun protection method, compared with $78.1 \%$ at baseline. Participants also reported significantly fewer sunburns at 2 years. Taken together, these findings suggest potential long-term benefits of melanoma genetic test reporting on adherence to recommended sun protection behaviors among unaffected members of high-risk families, with no decline in sun protection adherence following a negative test result.

Of course, because both unaffected $p 16$ mutation carriers and noncarriers alike reported improvements, it is difficult to attribute improvements among unaffected carriers to the receipt of a positive $p 16$ test result. Furthermore, in this study, the process of genetic testing and counseling were interconnected, with participants only receiving test results in conjunction with thorough education. Therefore, the effects of learning one's genetic status cannot be separated from the effects of the accompanying education. Future studies that incorporate similar education and counseling interventions with or without genetic testing are needed to fully delineate the impact of receiving genetic test results on prevention behavior. ${ }^{4}$

Regardless of whether the genetic test result ultimately accounted for the behavioral improvements reported, these long-term outcomes should allay concerns that members of high-risk families receiving negative p16 test results will decrease efforts to limit UVR exposure. ${ }^{10,11}$ Instead, unaffected noncarriers reported more frequent use of photoprotective clothing and increased practice of daily routine sun protection across the profile of recommended behaviors. Importantly, the reported frequency of all behaviors except sunscreen use corresponded to "fairly regular" use, ranging from 60 to $70 \%$ of the time, with the daily routine findings suggesting similarly consistent practice of photoprotective clothing use and UVR avoidance at 2 years.

\section{Implications for the prevention of sunburns}

Although daily routine sun protection was high at follow-up, reported sunburns were prevalent. Consistent with the findings of Glanz et al., ${ }^{21}$ more than $40 \%$ of participants reported one or more sunburns in the past 6 months at the 2-year follow-up. Our analysis of the reasons for sunburns suggested intervention targets for helping people who are largely compliant to avoid unintentional sunburns. Several participants became burned despite having applied sunscreen, because they had not applied it thoroughly or had not reapplied it after exercise or water activities. Thus, interventions that emphasize the "failure rate" of sunscreen and target the importance of frequent reapplication, especially during exercise or water activities, could be useful in preventing burns. Alternatively, protective clothing could be promoted as a more reliable method of protection for long periods outdoors or when water or perspiration may reduce the protection of sunscreen.

\section{Study limitations}

The primary limitation of this study is the modest sample size. Although we obtained a response rate of $64.9 \%$ over the 2 -year interval, the procedure of stratifying participants by mutation status and melanoma history to examine different profiles of sun protection behavior resulted in small subgroups for analysis. Therefore, these results await replication in a larger sample. Furthermore, although there were no differences between participants who completed the 2-year assessment and those who did not in baseline sun protection behavior or in such risk behaviors as sunburns, we cannot determine whether noncompleters were more or less compliant to sun protection recommendations at follow-up than those who provided follow-up data.

A second set of limitations involves generalizing study findings from this highly selected sample to other populations and settings. First, participants were recruited from two large kindreds and may have received support and encouragement from family members to maintain protective behaviors. It is possible that behavior change would not be as well sustained outside of a supportive family network or among families without a history of research participation. Second, nearly all participants were residents of Utah. Because of Utah's high elevation and sunny weather, Utah residents may have been especially sensitized to the need for consistent photoprotection. Therefore, additional studies in more diverse samples, in different family settings, in geographic regions outside the US Intermountain West, and among members of high-risk families with less prior research participation will be required to determine the generalizability of these findings.

It will be equally important to examine whether findings like these are replicated in typical clinical practice. The information provided in our research protocol is quite similar to information offered in clinical practice, as key elements of this protocol form the basis of GenoMel's international guide for incorporating melanoma genetic testing into clinical care. ${ }^{30}$ Participants received a single, detailed genetic counseling and prevention education session, without additional contact from the clinic (other than follow-up surveys) to motivate continued adherence. It will be important to conduct additional studies to understand the impact of tailored prevention education and counseling on prevention behaviors, even in the absence of genetic test results, ${ }^{31}$ and to determine whether more extensive patient contact might further improve prevention behaviors over time.

Third, as in any extended longitudinal study, multiple events may have occurred between the genetic counseling session and the 2-year follow-up that may have influenced prevention behavior. We examined prevention behaviors at 2 years among participants who reported a new melanoma or recurrence and among those who reported that a family member had been diagnosed with melanoma during the study. In neither case did these participants report greater sun protection behaviors than other participants in their respective participant groups. Another possibility is that medical care related to melanoma 
risk may affect adherence over time. For example, regular care by a dermatologist or other specialist may reinforce genetic counselors' preventive messages regarding sun protection and risk behaviors. With regard to this possibility, we note that $95 \%$ of $p 16$ mutation carriers and nearly $2 / 3$ of noncarriers reported having a medical professional who cared for their skin at some point in the 2-year interval. Of course, we cannot know if any counseling was provided during these visits that reinforced the prevention recommendations provided during genetic counseling. However, such intervening events as having affected relatives and seeking regular medical care are not necessarily limitations to the study, as they are likely common aspects of being a member of a high-risk melanoma-prone family and, in the case of more rigorous professional screening, a desired consequence of genetic counseling and test reporting. ${ }^{26}$

Finally, all measures were self-reported. Studies that employ objective measures of UVR exposure, corrected for the simultaneous practice of multiple photoprotection methods, are needed to definitively assess the impact of melanoma genetic test reporting on UVR exposure. . $^{32,33}$

\section{Conclusions}

Both unaffected carriers and unaffected noncarriers reported improvement in daily routine sun protection behavior 2 years following melanoma genetic test reporting, and affected carriers maintained high levels of adherence. Although these data cannot distinguish the effects of test reporting from the effects of the accompanying counseling, it is promising that daily routine sun protection increased, while the number of sunburns decreased. Importantly, there was no evidence suggesting decreased sun protection or increased risk behaviors among unaffected noncarrier family members following a negative test result. Overall, the present findings, along with prior findings concerning improvements among unaffected carriers in skin self-examination thoroughness and adherence to recommendations for annual professional total body skin examinations, ${ }^{17,21,26}$ indicate that genetic testing may motivate adherence to prevention and screening behavior in those found to carry a mutation. It will be an important task of future research to determine why personalized genetic information, paired with counseling, promotes sustained behavior change.

\section{ACKNOWLEDGMENTS}

This work was supported by a Funding Incentive Seed Grant, Office of the Vice President for Research, University of Utah, and a Cancer Control and Population Sciences Pilot Project Award from the Huntsman Cancer Institute awarded to L.G.A. and S.A.L. Support was also received from the Huntsman Cancer Foundation (HCF), the Tom C. Mathews, Jr. Familial Melanoma Research Clinic endowment, the Pedigree and Population Resource of Huntsman Cancer Institute, and the Utah Population Database. This research was supported by the Utah Cancer Registry, which is funded by contract N01-PC-35141 from the National Cancer Institute Surveillance, Epidemiology, and End Results (SEER) Program, with additional support from the Utah State Department of Health and the University of Utah. The authors acknowledge the use of core facilities supported by the National Institutes of Health through National Cancer Institute Cancer Center Support Grant 5P30CA420-14 awarded to Huntsman Cancer Institute, the genetic counseling core facility supported by the HCF, and National Center for Research Resources grant 1KL2RR025763-01 awarded to the University of Utah by the National Institutes of Health Office of the Director. The authors were supported in part in the preparation of this article by award number R01 CA158322-01 from the National Cancer Institute. The works of L.G.A. and S.A.L. are funded by the National Institutes of Health.

The content is solely the responsibility of the authors and does not necessarily represent the official views of the National Cancer Institute or the National Institutes of Health. The authors gratefully acknowledge the generous participation of all the family members in this study, without whom this project would not have been possible; Marybeth Hart, Erin Dola, and Lisa Wadge for their contributions to the development of the study; and Amber Kostial, Emily Bullough, Michelle Welch, Hoda Wali, Candace Larson, and Taylor Haskell for their service as study and/or clinic coordinators.

\section{DISCLOSURE}

S.A.L. serves on a Medical and Scientific Advisory Board for Myriad Genetics Laboratory, for which she has received an honorarium. She has collaborated with Myriad on a project to validate an assay that is unrelated to the research reported here. The other authors declare no conflict of interest.

\section{REFERENCES}

1. Botkin JR, Smith KR, Croyle RT, et al. Genetic testing for a BRCA1 mutation: prophylactic surgery and screening behavior in women 2 years post testing. Am J Med Genet A 2003;118A:201-209.

2. Aspinwall LG, Taber JM, Kohlmann W, Leachman SA. Psychological aspects of hereditary cancer risk counseling and genetic testing. In: Carr BI, Steel J (eds). Psychological Aspects of Cancer: A Guide to Emotional and Psychological Consequences of Cancer, Their Causes and Their Management. Springer: New York, 2013:31-64.

3. McBride CM, Bowen D, Brody LC, et al. Future health applications of genomics: priorities for communication, behavioral, and social sciences research. Am J Prev Med 2010;38:556-565.

4. Marteau TM, French DP, Griffin SJ, et al. Effects of communicating DNA-based disease risk estimates on risk-reducing behaviours. Cochrane Database Syst Rev 2010;10:CD007275.

5. Collins FS, Green ED, Guttmacher AE, Guyer MS; US National Human Genome Research Institute. A vision for the future of genomics research. Nature 2003;422:835-847.

6. Khoury MJ, Gwinn M, Yoon PW, Dowling N, Moore CA, Bradley L. The continuum of translation research in genomic medicine: how can we accelerate the appropriate integration of human genome discoveries into health care and disease prevention? Genet Med 2007;9:665-674.

7. Bishop DT, Demenais F, Goldstein AM, et al.; Melanoma Genetics Consortium Geographical variation in the penetrance of CDKN2A mutations for melanoma. J Natl Cancer Inst 2002;94:894-903.

8. Begg CB, Orlow I, Hummer AJ, et al.; Genes Environment and Melanoma Study Group. Lifetime risk of melanoma in CDKN2A mutation carriers in a populationbased sample. J Natl Cancer Inst 2005;97:1507-1515.

9. Leachman SA, Carucci J, Kohlmann W, et al. Selection criteria for genetic assessment of patients with familial melanoma. J Am Acad Dermatol 2009;61:677.e1-677.14.

10. Kefford R, Bishop JN, Tucker M, et al.; Melanoma Genetics Consortium. Genetic testing for melanoma. Lancet Oncol 2002;3:653-654 
11. Kefford RF, Mann GJ. Is there a role for genetic testing in patients with melanoma? Curr Opin Oncol 2003;15:157-161.

12. Aspinwall LG, Leaf SL, Kohlmann W, Dola ER, Leachman SA. Patterns of photoprotection following CDKN2A/p16 genetic test reporting and counseling. J Am Acad Dermatol 2009:60:745-757.

13. Bergenmar M, Brandberg Y. Sunbathing and sun-protection behaviors and attitudes of young Swedish adults with hereditary risk for malignant melanoma. Cancer Nurs 2001;24:341-350.

14. Brandberg $Y$, Jonell R, Broberg M, Sjödén PO, Rosdahl I. Sun-related behaviour in individuals with dysplastic naevus syndrome. Acta Derm Venereol 1996;76:381-384

15. Kasparian NA, Meiser B, Butow PN, Job RF, Mann GJ. Better the devil you know? High-risk individuals' anticipated psychological responses to genetic testing for melanoma susceptibility. J Genet Couns 2006;15:433-447.

16. Kasparian NA, Meiser B, Butow PN, Soames Job RF, Mann GJ. Anticipated uptake of genetic testing for familial melanoma in an Australian sample: An exploratory study. Psychooncology 2007;16:69-78.

17. Kasparian NA, Meiser B, Butow PN, Simpson JM, Mann GJ. Genetic testing for melanoma risk: a prospective cohort study of uptake and outcomes among Australian families. Genet Med 2009;11:265-278.

18. Bränström R, Kasparian NA, Affleck $P$, et al. Perceptions of genetic research and testing among members of families with an increased risk of malignant melanoma. Eur J Cancer 2012;48:3052-3062.

19. Taber JM, Aspinwall LG, Kohlmann W, Dow R, Leachman SA. Parental preferences for CDKN2A/p16 testing of minors. Genet Med 2010;12:823838.

20. Christensen KD, Roberts JS, Shalowitz DI, et al. Disclosing individual CDKN2A research results to melanoma survivors: interest, impact, and demands on researchers. Cancer Epidemiol Biomarkers Prev 2011;20:522-529.

21. Glanz K, Volpicelli K, Kanetsky PA, et al. Melanoma genetic testing, counseling, and adherence to skin cancer prevention and detection behaviors. Cancer Epidemiol Biomarkers Prev 2013;22:607-614.

22. Cannon-Albright LA, Goldgar DE, Meyer LJ, et al. Assignment of a locus for familial melanoma, MLM, to chromosome 9p13-p22. Science 1992;258:11481152.
23. Kamb A, Shattuck-Eidens D, Eeles $R$, et al. Analysis of the $p 16$ gene (CDKN2) as a candidate for the chromosome $9 p$ melanoma susceptibility locus. Nat Genet 1994;8:23-26.

24. Florell SR, Boucher KM, Garibotti G, et al. Population-based analysis of prognostic factors and survival in familial melanoma. J Clin Oncol 2005;23:7168-7177.

25. Aspinwall LG, Leaf SL, Dola ER, Kohlmann W, Leachman SA. CDKN2A/p16 genetic test reporting improves early detection intentions and practices in highrisk melanoma families. Cancer Epidemiol Biomarkers Prev 2008;17:1510-1519.

26. Aspinwall LG, Taber JM, Leaf SL, Kohlmann W, Leachman SA. Melanoma genetic counseling and test reporting improve screening adherence among unaffected carriers 2 years later. Cancer Epidemiol Biomarkers Prev 2013;22:1687-1697.

27. Aspinwall LG, Taber JM, Leaf SL, Kohlmann W, Leachman SA. Genetic testing for hereditary melanoma and pancreatic cancer: a longitudinal study of psychological outcome. Psychooncology 2013;22:276-289.

28. Gandini S, Sera F, Cattaruzza MS, et al. Meta-analysis of risk factors for cutaneous melanoma: II. Sun exposure. Eur J Cancer 2005;41:45-60.

29. Steffen $A D$, Glanz K, Wilkens LR. Identifying latent classes of adults at risk for skin cancer based on constitutional risk and sun protection behavior. Cancer Epidemiol Biomarkers Prev 2007;16:1422-1427.

30. GenoMEL the Melanoma Genetics Consortium. Genetic Counselling and Testing for Hereditary Melanoma. 2011. http://www.genomel.org/genetic counselling/. Accessed 14 March 2014

31. Albada A, Ausems MG, Bensing JM, van Dulmen S. Tailored information about cancer risk and screening: a systematic review. Patient Educ Couns 2009;77:155-171

32. Glanz K, Mayer JA. Reducing ultraviolet radiation exposure to prevent skin cancer: methodology and measurement. Am J Prev Med 2005;29: 131-142

33. Stump TK, Aspinwall LG, Leachman SA, Taber JM, Edwards R. Protectionadjusted length of exposure (PALE): a new self-report measure of UVR exposure that captures multiple modalities of photoprotection. June 2013: Paper presented at the annual meeting of the Melanoma Genetics Consortium, Philadelphia, PA. 